INPLASY

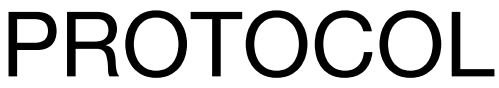

To cite: Infantes-Paniagua et al. Effects of physical activity breaks on the attention of students: A systematic review with meta-analysis. Inplasy protocol 202110054. doi: 10.37766/inplasy2021.1.0054

Received: 15 January 2021

Published: 16 January 2021

Corresponding author:

Filipe Manuel Clemente

filipe.clemente5@gmail.com

Author Affiliation:

Escola Superior Desporto e Lazer, Instituto Politécnico de Viana do Castelo, Viana do Castelo, Portugal

Support: None.

Review Stage at time of this submission: Formal screening of search results against eligibility criteria.

Conflicts of interest: None.

\section{Effects of physical activity breaks on the attention of students: A systematic review with meta-analysis}

Infantes-Paniagua, $A^{1}$; Silva, $\mathrm{AF}^{2}$; Ramirez-Campillo, $\mathrm{R}^{3}$; Sarmento, H4; Fernández, FTG5; González-Víllora, S6; Clemente, $\mathrm{FM}^{7}$.

Review question / Objective: This systematic review with meta-analysis was conducted to compare the effects of physical activity breaks vs. control conditions in the attention of students.

Condition being studied: Attention of students subjected to physical activity breaks.

Information sources: Electronic databases (PubMed, PsycINFO, Scopus, SPORTDiscus and Web of Science) were searched for relevant publications prior to the 12 January 2021.

INPLASY registration number: This protocol was registered with the International Platform of Registered Systematic Review and Meta-Analysis Protocols (INPLASY) on 16 January 2021 and was last updated on 16 January 2021 (registration number INPLASY202110054).

\section{INTRODUCTION}

Review question / Objective: This systematic review with meta-analysis was conducted to compare the effects of physical activity breaks vs. control conditions in the attention of students.

Rationale: Active breaks (ABs) are currently getting attention within educational context
(Daly-Smith et al., 2018). ABs consist of short periods of classroom-based physical activity (PA), usually between five and 15 minutes (Masini et al., 2019), which are placed into a class routine (Daly-Smith et al., 2018; Masini et al., 2019; Watson et al., 2017). These can be implemented by the teacher (Masini et al., 2019) during academic instructions or between them (Goh et al., 2016). Compared to other kinds 
of school-based PA interventions, ABs show some advantages like (i) $A B s$ do not require special spaces or equipment, (ii) teachers can choose the moments when introducing the $A B s$ according to their lessons' necessities (Masini et al. 2019), and (iii) these are not too much timeconsuming for real school practice (Van den Berg et al., 2017).

Condition being studied: Attention of students subjected to physical activity breaks.

\section{METHODS}

Search strategy: Keywords and synonyms were entered in various combinations in all fields: ("activ* break*" OR "physical break" OR "physical activity break" OR "exercise break" OR "brain break" OR "brain hacking" OR "movement learning" OR "active learning") AND (student* OR class* OR school*) AND attent*.

Participant or population: Students from any age or sex, healthy and with no mental diseases diagnosed.

Intervention: Physical activity breaks consisting in short periods of exercise in class during or between academic lessons.

Comparator: Control conditions (passive or non-active breaks, with a small amount of physical activity).

Study designs to be included: Counterbalanced cross-over design; RCT or non-RCT.

Eligibility criteria: Inclusion criteria: (1) Students from any age or sex, healthy and with no mental diseases diagnosed; (2) Physical activity breaks consisting in short periods of exercise in class during or between academic lessons (e.g., structured exercises, free exercise). (3) Control conditions (passive or non-active breaks, with a small amount of physical activity). (4) Attentional outcomes (e.g. focused or selective attention, vigilance, inhibitory control, etc.) measured before (pre-) and after (post-) activity breaks or a chronic intervention of active breaks.; (5) Counterbalanced cross-over design (randomized and non-randomized can be included, since all revealing no significant differences in control conditions).; (6) Only original and full-text studies written in English. Exclusion criteria: (1) Other populations than students (e.g., workers, athletes).; (2) No physical activity breaks (e.g., physical education classes; playing with instruments without allowing PA).; (3) Other forms of physical activity interventions (e.g., physical education lessons).; (4) Other outcomes than attention; No pre-post comparison.; (5) Other study designs that do not allow comparisons within-subjects for the two conditions (control and ABs).; (6) Written in other language than English. Other article types than original (e.g., reviews, letters to editors, trial registrations, proposals for protocols, editorials, book chapters and conference abstracts).

Information sources: Electronic databases ( $P$ u b M ed, Ps y c I N O, Scopus, SPORTDiscus and Web of Science) were searched for relevant publications prior to the 12 January 2021.

Main outcome(s): Aiming to establish consistency in data analyzing and reporting, only measures that were analyzed pre- and post-active breaks were included. Several outcomes were considered for extraction as long as these were a measure of attention: (i) focused or selective attention, (ii) vigilance or sustained attention, and (iii) inhibitory control, among others.

Data management: A data extraction was prepared in Microsoft Excel sheet (Microsoft Corporation, Readmon, WA, USA) in accordance with the Cochrane Consumers and Communication Review Group's data extraction template (Group, 2016). The Excel sheet was used to assess inclusion requirements and subsequently tested for all selected studies.

Quality assessment / Risk of bias analysis: A priori, the Strengthening the Reporting of Observational Studies in Epidemiology 
(STROBE) statement purposed by Von Elm et al. (2007) will be used, although depending on the characteristics of all included studies a more approriate tool could be employed.

Strategy of data synthesis: Analysis and interpretation of results in this systematic review and meta-analysis were only conducted in the case of at least three study groups provided baseline and physical active breaks-related data for the same measure.

Subgroup analysis: Time of exercise; sex; age.

Sensibility analysis: The risk of bias was explored using the extended Egger's test (Egger et al., 1997). To adjust for publication bias, a sensitivity analysis was conducted using the trim and fill method (Duval and Tweedie, 2000), with LO as the default estimator for the number of missing studies (Shi and Lin, 2019).

Language: English.

Country(ies) involved: Portugal, Spain, Chile.

Keywords: Attention; active breaks; physical activity; performance.

Contributions of each author:

Author 1 - Álvaro Infantes-Paniagua - Lead the systematic review, conducted the search, methodological assessment, data synthesis, wrote and revised the manuscript.

Author 2 - Ana Filipa Silva - Wrote and revised the manuscript.

Author 3 - Rodrigo Ramirez-Campillo Performed the statistical analysis and report. Wrote and revised the manuscript.

Author 4 - Hugo Sarmento - Performed the search and screening. Wrote and revised the manuscript.

Author 5 - Francisco Tomás González Fernández - Conducted the search, methodological assessment, data synthesis, wrote and revised the manuscript.
Author 6 - Sixto González-Víllora - Wrote and revised the manuscript.

Author 7 - Filipe Manuel Clemente - Wrote and revised the manuscript.

Conflicts of interest: Álvaro InfantesPaniagua, Ana Filipa Silva, Rodrigo Ramirez-Campillo, Hugo Sarmento, Francisco Tomás González Fernández, Sixto González-Víllora, Filipe Manuel Clemente declare that they have no conflicts of interest relevant to the content of this review. 